\title{
Transport barrier created by dimerized islands ${ }^{\hbar}$
}

\author{
M. Roberto ${ }^{\mathrm{a}}$, E.C. da Silva ${ }^{\mathrm{b}}$, I.L. Caldas ${ }^{\mathrm{b}}$, R.L. Viana ${ }^{\mathrm{c}, *}$ \\ ${ }^{a}$ Departamento de Física, Instituto Tecnológico de Aeronáutica, Centro Técnico Aeroespacial, \\ 12228-900, São José dos Campos, São Paulo, Brazil \\ bInstituto de Física, Universidade de São Paulo, 05315-970, São Paulo, São Paulo, Brazil \\ ${ }^{\mathrm{c}}$ Departamento de Física, Universidade Federal do Paraná, 81531-990, Curitiba, Paraná, Brazil
}

Received 31 October 2003; received in revised form 21 January 2004

Available online 18 May 2004

\begin{abstract}
We investigated the structure of magnetic field lines in a tokamak with reversed magnetic shear and non-symmetric perturbations by means of analytically derived area-preserving non-twist Poincaré maps. The maps are used to study magnetic island dimerization and the formation of a transport barrier in the chaotic layer of field lines.

(c) 2004 Elsevier B.V. All rights reserved.
\end{abstract}

PACS: 52.55.Fa; 52.55.Dy; 52.25.Gj

Keywords: Non-twist maps; Reconnection; Magnetic field lines

Over the last 40 years, area preserving maps have been used to describe magnetic field line behavior in fusion plasma confinement schemes like tokamaks [1]. Plasma particles in a tokamak are confined in the toroidal chamber by the combination of two basic magnetic fields in the directions of the major and minor curvatures of the torus. The superposition of these basic fields produces magnetic field lines with helical shape, lying on constant pressure surfaces called magnetic, or flux surfaces, with topology of nested tori [2]. The magnetic field line equations, after proper parametrization (the time-like variable is an ignorable coordinate), can be viewed as Hamilton's equation describing an integrable system, where phase space trajectories are identified with magnetic field lines [3]. If the torus is intersected by a Poincaré surface of section we can, at least in principle, write down an area preserving canonical mapping relating

\footnotetext{
This work was made possible with partial financial support of the following agencies: CNPq and FAPESP.

${ }^{*}$ Corresponding author.

E-mail address: viana@fisica.ufpr.br (R.L. Viana).
} 


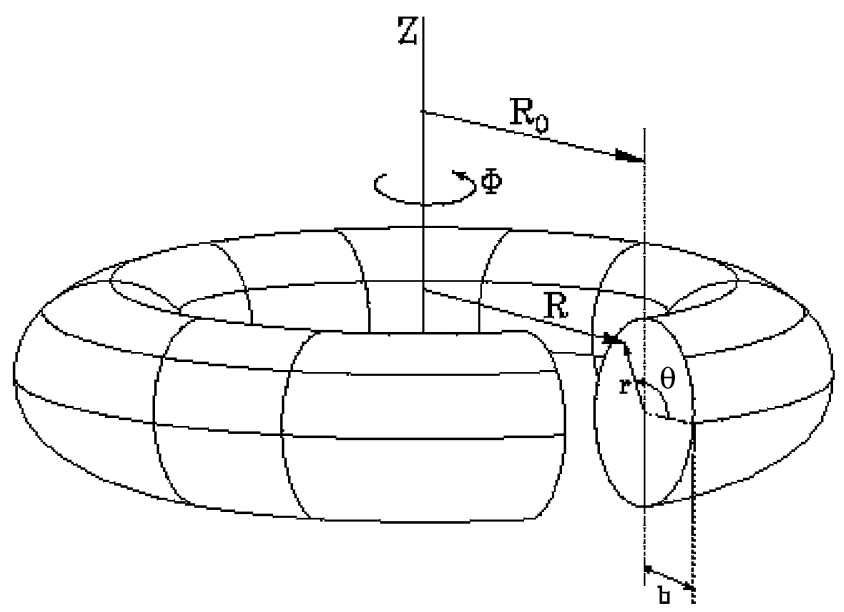

Fig. 1. Basic geometry of the tokamak.

the coordinates of a field line at a given time in terms of the same coordinates at a previous time.

The use of two-dimensional symplectic maps enables us to harness the powerful methods of Hamiltonian dynamics in order to explain the observed changes in the magnetic field structure due to magnetostatic perturbations [4]. One such result is the creation of a chaotic magnetic field line layer in the plasma due to the resonant perturbing fields in tokamaks. Here the word chaos must be intended in the Lagrangean sense: two field lines, very close to each other, depart at an exponential rate as we follow their revolutions along the toroidal chamber [5].

A situation in which the presence of magnetic field line chaos can help plasma confinement is the creation of a transport barrier to reduce particle escape in tokamaks. It has been recently observed that this may occur if there is a negative magnetic shear region within the plasma column [6]. Unlike most area preserving maps used to investigate field line behavior, negative shear configurations are best described by non-twist area-preserving maps [3,7], which does not satisfy the non-degeneracy condition for the Kolmogorov-Arnold-Moser (KAM) theorem to be valid [8]. The transport barrier arises from a combination of typical features of non-twist maps: reconnection and bifurcation, occurring in the reversed shear region [9]. This barrier is embedded in a chaotic field line region in the tokamak peripheral region, and which can be generated, for example, by external magnetostatic perturbations [10].

In this paper, we will derive an area-preserving map by considering the superposition of a non-integrable field on the tokamak equilibrium field with a negative shear region. This map can be used to numerically evidence the formation of a transport barrier due to a reconnection-bifurcation mechanism.

Fig. 1 presents a schematic picture of a tokamak, showing the basic coordinates used to locate a magnetic field line. The $Z$-coordinate runs along the major axis of the 

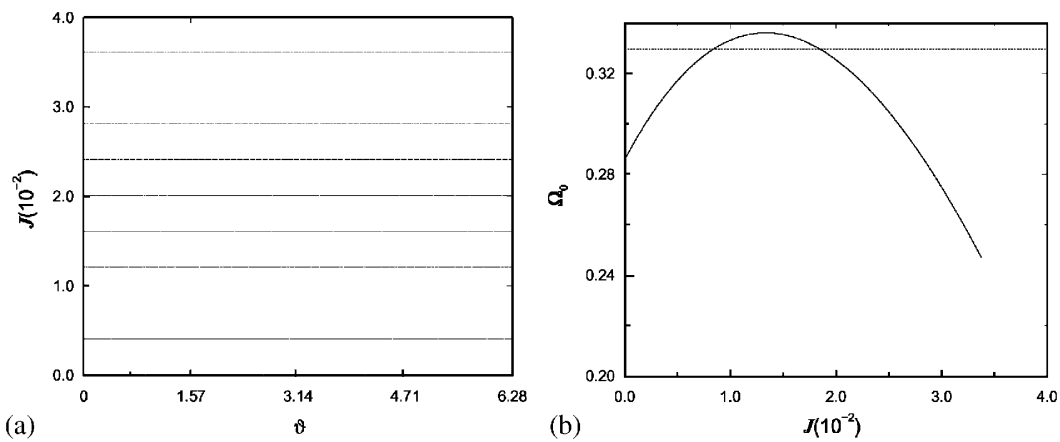

Fig. 2. (a) Poincaré map (in dimensionless action-angle variables) for the equilibrium tokamak field; (b) frequency profile for the equilibrium field. The dotted line represents the location of a rational surface with frequency $\frac{1}{3}$.

tokamak, with the corresponding cylindrical coordinates $(R, \Phi)$. The Poincaré surface of section is taken at a fixed value of the toroidal angle $\Phi$, and the field line coordinates at this section $(r, \theta)$ are counted from the minor axis of the torus, located at $R=R_{0}$. We thus have $R=R_{0}+r \cos \theta$, and $r=b$ defines the tokamak boundary. The field line equations in this coordinate system are $\left(\mathrm{d} r / B_{r}\right)=\left(r \mathrm{~d} \theta / B_{\theta}\right)=\left(\mathrm{d} \Phi / B_{\Phi}\right)$, where $\mathbf{B}=$ $\left(B_{r}, B_{\theta}, B_{\Phi}\right)$ is the tokamak magnetic field [2].

Thanks to the $\nabla \cdot \mathbf{B}=0$ condition this can be shown to be a hamiltonian system with one degree of freedom [3]. It is integrable provided the equilibrium field components do not depend explicitly on the time-like variable $\Phi$. However, the field line coordinates $(r, \theta, \Phi)$ are not proper canonical coordinates, and we must previously define action-angle variables $(J, \vartheta)$ for this system. The details of the derivation can be found in Ref. [10], but the action-angle variables $(J, \vartheta)$ are related to the $(r, \theta)$ coordinates, respectively. Now the magnetic field line equations can be cast in a Hamiltonian form

$$
\frac{\mathrm{d} J}{\mathrm{~d} t}=-\frac{\partial H_{0}}{\partial \vartheta}, \quad \frac{\mathrm{d} \vartheta}{\mathrm{d} t}=\frac{\partial H_{0}}{\partial J}
$$

where $t=\Phi$ and $H_{0}$ is the equilibrium field line hamiltonian. Since it represents an integrable system, $H_{0}$ depends only on $J$, hence the above equations are readily integrated to furnish [see Fig. 2(a)] $J(t)=J(0)=$ const., and $\vartheta(t)=\vartheta(0)+\Omega_{0}(J) t$, where $\Omega_{0} \equiv \mathrm{d} H_{0} / \mathrm{d} J$ is the frequency related to the field line revolutions along the torus. The latter quantity turns out to be a function of the equilibrium magnetic field $\mathbf{B}$, and thus reflects the details of the plasma current profile. In this paper we consider a plasma with the so-called reversed shear region, for which the frequency profile is non-monotonic, as shown by Fig. 2(b).

The field line configuration can be turned out non-integrable by adding a symmetry breaking perturbation, like that caused by the $\Phi$-dependent magnetic field of external currents on a coil, at a given toroidal section of the tokamak torus. We can model this effect by a hamiltonian perturbation which is periodic in both $\vartheta$ and $t$ variables, so 
that the field line hamiltonian is written as

$$
H(J, \vartheta, t)=H_{0}(J)+\varepsilon H_{m_{0}}^{*}(J) \cos \left(m_{0} \vartheta-n_{0} t\right) \sum_{k=-\infty}^{+\infty} \delta\left(t-k \frac{2 \pi}{N_{r}}\right),
$$

where $\varepsilon$ represents the perturbation strength, which is proportional to the current applied in the external conductors. If $\varepsilon \ll 1$ we can regard this as a near-integrable system with $1+\frac{1}{2}$ degrees of freedom. The $\Phi$-dependence of the perturbation is represented by a periodic sequence of delta kicks at $\Phi=2 k \pi / N_{r}$ with $k=0,1,2, \ldots$.

The perturbation resonates with the equilibrium field causing the destruction of the invariant surfaces $J=J^{*}$ for which the frequency $\Omega_{0}$ is a rational number $\Omega_{0} \equiv$ $m_{0} / n_{0}$, according to the Poincaré-Birkhoff theorem [4]. Since the frequency profile is non-monotonic, there are two such surfaces for certain values of $\Omega_{0}$, as illustrated in Fig. 2(b) for $\Omega_{0}=\frac{1}{3}$. This fact also accounts for the failing of the unperturbed Hamiltonian $H_{0}$ to satisfy KAM theorem. Hence, the field line mapping belongs to a class of non-twist maps, which have been extensively studied in the literature [3]. One of the consequences arising from the non-twist condition is that the Poincare-Birkhoff islands are not pendular (i.e., their widths do not increase as the square root of $\varepsilon$ ), and we need a higher order approximation in order to capture the essentials of the twins islands' behavior.

After dropping the constant terms, we expand the Hamiltonian (2) to third order in $\Delta J=J-J^{*}$, and perform a canonical transformation to new action-angle variables $\left(J^{\prime}, \vartheta^{\prime}\right)$ to eliminate the explicit time-dependence, what can be done through the generating function $S\left(J^{\prime}, \vartheta, t\right)=\left[\vartheta-\left(n_{0} t / m_{0}\right)\right] J^{\prime}$, leading to the local Hamiltonian which, after rescaling, is

$$
H\left(J^{\prime}, \vartheta^{\prime}\right)=\frac{1}{2} J^{\prime 2}-\frac{\alpha}{3} J^{\prime 3}+\kappa \cos \left(m_{0} \vartheta^{\prime}\right) \sum_{k=-\infty}^{+\infty} \delta\left(t-k \frac{2 \pi}{N_{r}}\right),
$$

where $\alpha=W / M$ and $\kappa=K / M$, with

$$
\left.M\left(J^{*}\right) \equiv \frac{\mathrm{d} \Omega_{0}}{\mathrm{~d} J}\right|_{J=J^{*}},\left.\quad W\left(J^{*}\right) \equiv \frac{1}{2} \frac{\mathrm{d}^{2} \Omega_{0}}{\mathrm{~d} J^{2}}\right|_{J=J^{*}}, \quad K\left(J^{*}\right) \equiv \varepsilon H_{m_{0}}^{*}\left(J_{1,2}^{*}\right) .
$$

In the $\alpha=0$ limit, the Hamiltonian above reduces to that of a nonlinear pendulum, which is the standard procedure used in perturbation theory to describe the phase-space structure near a given resonance [4]. Hence, $\alpha$ measures, so as to speak, the non-pendular character of the island chains. The fact that the "time"-dependence of the Hamilton equations is in the form of a periodic sequence of delta function kicks enables us to define discretized variables $\left(J_{k}^{\prime}, \vartheta_{k}^{\prime}\right)$, as the angle and action, respectively, just after the $k$ th kick, which occurs at time $t_{k}=\left(2 k \pi / N_{r} m_{0}\right)$, hence $t_{k+1}=t_{k}+\left(2 \pi / m_{0} N_{r}\right)$. The resulting field line map is thus

$$
J_{k+1}^{\prime}=J_{k}^{\prime}+m_{0} \kappa \sin \left(m_{0} \vartheta_{k+1}^{\prime}\right), \quad \vartheta_{k+1}^{\prime}=\vartheta_{k}^{\prime}+\frac{2 \pi}{N_{r}} J_{k}^{\prime}\left(1-\alpha J_{k}^{\prime}\right),
$$

where $\vartheta^{\prime}$ is to be taken modulo $2 \pi$.

Fig. 3 shows a sequence of three Poincaré maps, obtained for different perturbation strengths, which describe the evolution of the nonpendular islands towards reconnection. 

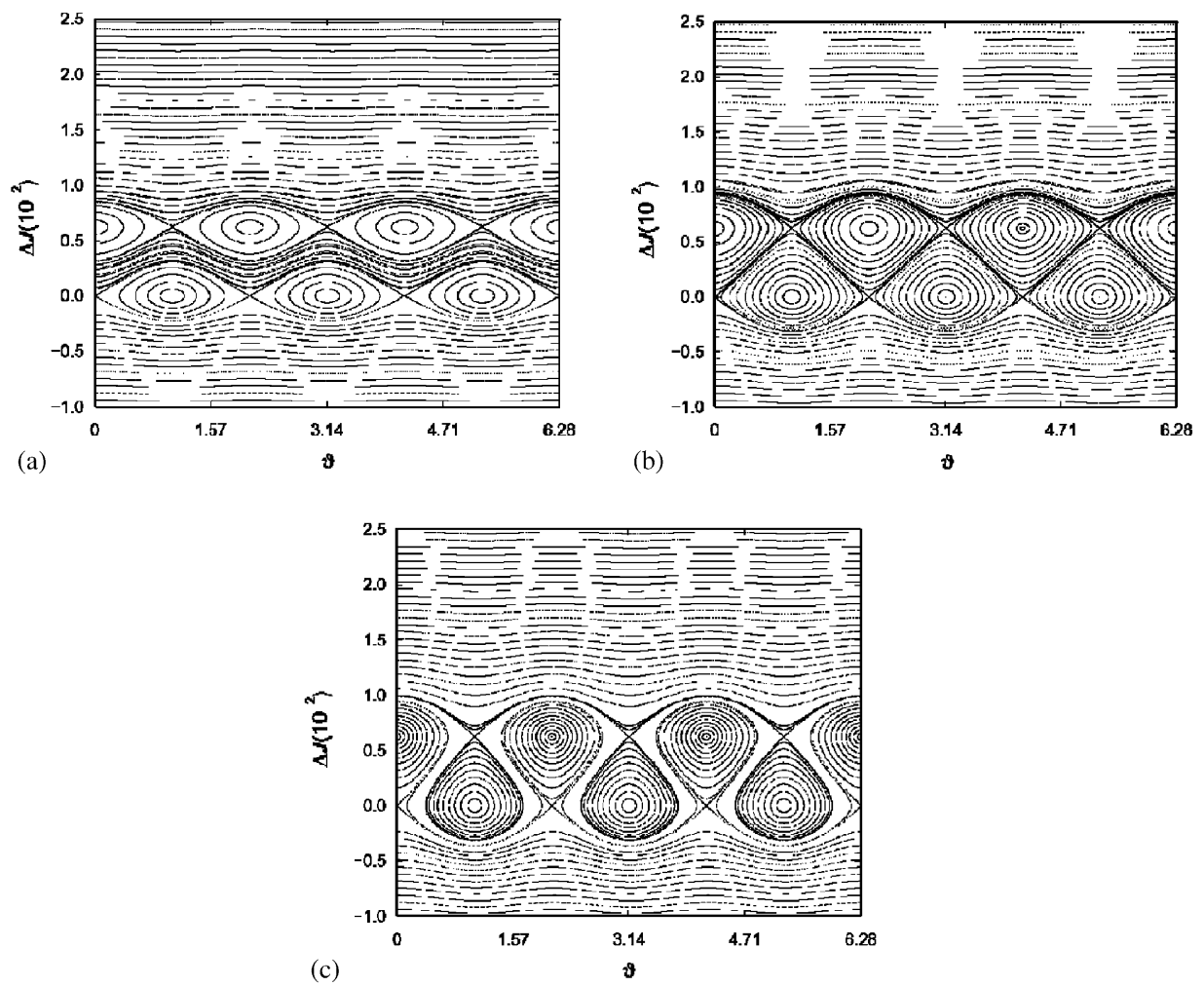

Fig. 3. Poincaré maps for one resonant mode with (a) $\kappa=4.221 \times 10^{-4}$; (b) $\kappa=1.842 \times 10^{-3}$; (c) $\kappa=3.799 \times 10^{-3}$.

For weak perturbations [Fig. 3(a)] we have two chains of non-pendular islands centered at the points $J_{1}^{*}$ and $J_{2}^{*}$ given by the intersections of the frequency profile with the line $\Omega_{0}=\frac{1}{3}$ in Fig. 2(b). Observe that in the vicinity of the island separatrices there are no visible chaotic layers, as expected from the non-KAM nature of the Hamiltonian. Hence, the islands can approach each other without destruction of tori. For a perturbation strong enough [Fig. 3(b)] the island chains dimerize, forming a unique sequence of islands which share a common separatrix. This is called reconnection, but it is rather a topological effect, involving no dissipative breakup and re-wiring of the field lines, as it occurs in astrophysical and fusion situations [11]. After the reconnection takes place [Fig. 3(c)] we find curves which encircle both sets of islands, forming a unique path which will be the foundation of the transport barrier to be described next.

The island reconnection process we have studied does not directly lead to a transport barrier since the chaotic region which exists due to lack of integrability is too tiny to have noticeable effect on the overall field line behavior. The transport barrier described in Ref. [9] results from the existence of a chaotic region into which a barrier is embedded. The case of just one resonant mode does not allow for such a wide chaotic 

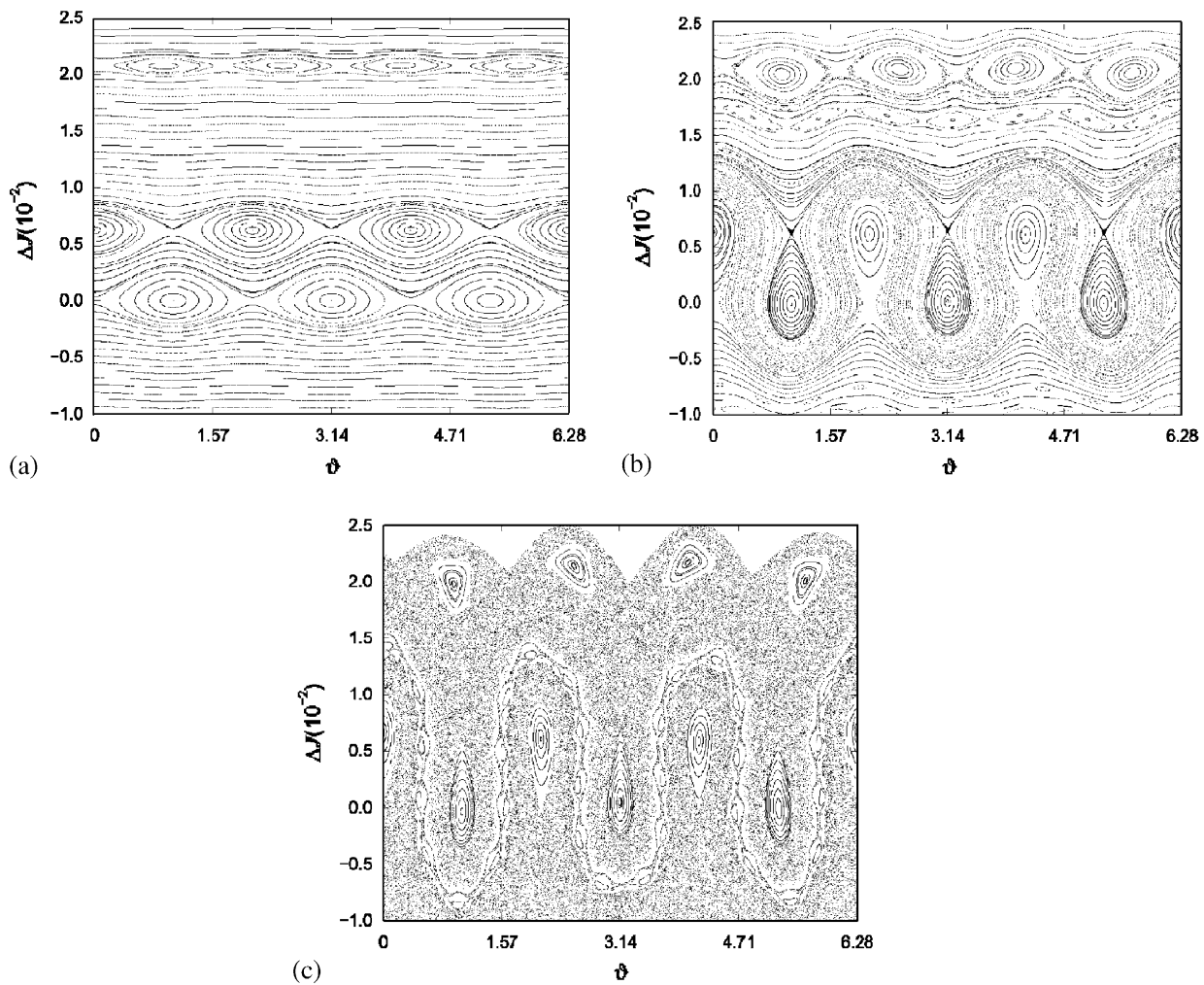

Fig. 4. Poincaré maps for two resonant modes with (a) $\kappa=1.149 \times 10^{-3}, \tilde{\kappa}=-5.288 \times 10^{-4}$; (b) $\kappa=6.078 \times 10^{-2}, \tilde{\kappa}=-2.797 \times 10^{-2} ;$ (c) $\kappa=4.323 \times 10^{-1}, \tilde{\kappa}=-1.989 \times 10^{-1}$.

layer, such that we need to consider at least two resonant modes to cope for this effect. If the main resonant mode has mode numbers $\left(m_{0}, n_{0}\right)$, the second mode has $\left(m_{0}+1, n_{0}\right)$.

This second resonant mode produces a chain of magnetic islands with different topological characteristics since it comes from a different (and unique) resonant surface. Hence it will not dimerize with the first resonant mode. As the perturbation strength increases both islands become wider and they interact as predicted by standard global stochasticity mechanisms [4]. If we pick up two resonant modes from the Hamiltonian (3), there will appear terms in $\cos \left(m_{0} \vartheta^{\prime}\right)$ and $\cos \left[\left(m_{0}+1\right) \vartheta^{\prime}\right]$. The derivation of the field line map is entirely similar to that described in the previous section, and furnishes

$$
\begin{aligned}
& J_{k+1}^{\prime}=J_{k}^{\prime}+m_{0} \kappa \sin \left(m_{0} \vartheta_{k+1}^{\prime}\right)+\left(m_{0}+1\right) \tilde{\kappa} \sin \left[\left(m_{0}+1\right) \vartheta_{k+1}^{\prime}\right], \\
& \vartheta_{k+1}^{\prime}=\vartheta_{k}^{\prime}+\frac{2 \pi}{N_{r}} J_{k}^{\prime}\left(1-\alpha J_{k}^{\prime}\right),
\end{aligned}
$$

where $\tilde{\kappa}$ is the amplitude of the second resonant mode. 
Fig. 4 shows, in the Poincare surface of section, the evolution of two resonant modes as the perturbation strengths $\kappa$ and $\tilde{\kappa}$ increase. The second resonant mode, albeit existing for any non-zero perturbation strength, is noticeable only for higher values of $\kappa$ and $\tilde{\kappa}$ than those previously considered. In order to have non-dimerized islands for such strong perturbations, it has been necessary to choose other mode amplitudes. Fig. 4(a) exemplifies a case where a second mode coexists with non-dimerized twin chains of islands corresponding to the first resonant mode. As the perturbation strength increases further [Figs. 4(b) and (c)] we can see that: (i) the twin chains of the first mode have already dimerized and suffer reconnection, with open surfaces exploring the former twin chains (this is actually necessary for the transport barrier be present); and (ii) there are visible chaotic layers surrounding both sets of islands.

Higher perturbation strengths would destroy entirely the invariant curves between both chains and a wide chaotic layer appears surrounding both modes. The transport barrier appears embedded in this large chaotic layer and is structured around the path of open curves which encircled the formerly dimerized islands after reconnection. This layer acts as an effective transport barrier since it hampers the radial diffusion of field lines, and arises from the "stickiness" effect that the region near the former separatrices of islands exert on the chaotic trajectories. The effect of this barrier on field line transport can be inferred from the study of field line diffusion by using the obtained map [11].

\section{References}

[1] B. Chirikov, Phys. Rep. 52 (1979) 265.

[2] J. Wesson, Tokamaks, Cambridge University Press, Cambridge, 1987.

[3] P.J. Morrison, Phys. Plasmas 7 (2000) 2279.

[4] A.J. Lichtenberg, M.A. Lieberman, Regular and Chaotic Dynamics, 2nd Edition, Springer, New York, Berlin, Heidelberg, 1992.

[5] E. Ott, Chaos in Dynamical Systems, Cambridge University Press, Cambridge, 1994.

[6] E. Mazzucato, S.H. Batha, M. Beer, et al., Phys. Rev. Lett. 77 (1996) 3145.

[7] R. Balescu, Phys. Rev. E 58 (1998) 3781.

[8] D. Castillo-Negrete, J.M. Greene, P.J. Morrison, Physica D 91 (1996) 1.

[9] G. Corso, F.B. Rizzato, Phys. Rev. E 58 (1998) 8013.

[10] E.C. da Silva, I.L. Caldas, R.L. Viana, IEEE Trans. Plasma Sci. 29 (2001) 617.

[11] M. Roberto, E.C. da Silva, I.L. Caldas, R.L. Viana, Phys. Plasmas 11 (2004) 214. 